\title{
Ab Initio Formation of Galaxies, Groups and Large-Scale Structure
}

\author{
Antonaldo Diaferio \\ Max-Planck-Institut für Astrophysik, Karl-Schwarzschild-Str. 1, \\ D.85740 Garching, Germany
}

\begin{abstract}
For the first time, the combination of semi-analytic modelling of galaxy formation and $N$-body simulations of cosmic structure formation enables us to model, at the same time, both the photometric and the clustering properties of galaxies. Two Cold Dark Matter Universes provide a reasonable fit to the observed properties of galaxies, groups and clusters, including luminosities, colours, density and velocity biases. We show how the properties of galaxies and groups on small scales are inextricably connected with the global properties of the Universe.
\end{abstract}

\section{Introduction}

Groups of galaxies probe the intermediate scale between galaxies and clusters. A satisfactory theory for the formation and the evolution of these systems has always been difficult, because the size of groups is small enough to require the modelling of the properties and the internal structure of the galaxy members and, at the same time, large enough to require the modelling of the large scale structure sorrounding the groups.

For the last twenty years, $N$-body simulations have modelled groups with vacuum boundary conditions in order to resolve the internal kinematics of the galaxies (e.g. Carnevali et al. 1981; Barnes 1985; Diaferio et al. 1993; Athanassoula et al. 1997). Large-scale structure simulations have provided cosmological boundary conditions without resolving the galaxy internal properties (e.g. Frederic 1995a,b; Nolthenius et al. 1997). Both sets of simulations have not modelled the physics of galaxy formation and evolution which provides observable quantities, such as luminosity and colour. However, modelling groups within a cosmological context with sufficient resolution is extremely important, because groups both trace the large-scale structure of the Universe (e.g. Ramella et al. 1997, 1999) and are the sites of galaxy interactions (Hickson 1997; but see also e.g. Bettoni; Bosma; Rampazzo; Temporin, these proceedings). Galaxy interactions are connected with the formation of AGN's (Byrd, these proceedings), quasars (Cavaliere, these proceedings) and multiple central black holes (Valtonen, these proceedings). The importance of this large--small scale connection has been particularly important for successfully modelling the very existence of compact groups (Mamon 1986, 1989; Diaferio et al. 1994; Hernquist et al. 1995). The enormous progress of the last few years in probing the high-redshift Universe, which shows a high degree of galaxy clustering (see e.g. Mazure \& 
Le Fèvre 1999), impels us to build a cosmologically motivated theory of galaxy formation.

State-of-the-art $N$-body/hydrodynamic simulations have started only recently to barely reach the resolution required to follow the formation of galaxies within a cosmological context, even though only some of the relevant physical processes are included (Pearce et al. 1999). For the time being, a more fruitful approach has been the combination of semi-analytic modelling of galaxy formation with $N$-body simulations. The semi-analytic approach enables us to have full control of the relevant galaxy formation processes, which occur on par$\mathrm{sec}$ or smaller scales, namely star formation, stellar evolution, and effects from supernova explosions. The $N$-body simulations yield the merging history of galaxies and their phase space position, which are the result of group and cluster formation occurring on megaparsec and larger scales. By combining the two techniques, we are able to connect the stellar population properties of galaxies with their clustering and kinematic properties. This approach has already been particularly successful (Kauffmann et al. 1999a,b; Diaferio et al. 1999).

Here, I briefly review this technique (Section 2). I then show some remarkable results which the self-consistency of the approach yields automatically: the two component luminosity functions of groups (Section 3) and the density and velocity biases of cluster galaxies with differing colours (Section 4). Extracting mock redshift surveys from the simulation box also allows a direct comparison with the observed large-scale distribution of galaxies (Section 5). These results show the effectiveness of our approach, and also the partial success of the cosmological models.

\section{The Galaxy Formation Recipe}

According to standard inflationary models (e.g. Peacock 1999), large scale structure in the present day Universe originates from primordial density perturbations amplified by gravitational instability. These models also predict that most of the mass in the Universe is non-baryonic and therefore "dark". Assuming a Cold Dark Matter (CDM) power spectrum of the perturbations (e.g. Efstathiou et al. 1992), the clustering proceeds hierarchically: large perturbations collapse first, forming small dark matter halos which aggregate to form larger halos. Within these halos the baryonic gas cools, flows into the centre of the halo, and forms stars which evolve and, eventually, some of them explode as supernovae. The supernovae enrich the interstellar gas with metals and reheat part of the gas.

We need two basic ingredients to implement this picture: (1) the merging history of dark matter halos; and (2) recipes for the relevant processes involving the baryonic matter. Traditional semi-analytic models (e.g. Somerville \& Primack 1999 and references therein) use Monte Carlo simulations based on the extended Press \& Schechter (1974) formalism (e.g. Bower 1991) to derive the merging tree of dark matter halos. Here, instead, we derive the merger trees from $N$-body simulations. Moreover, we identify the galaxy harbored by the halo with the central dark matter particle of the halo: the galaxy and the central particle share the same phase space coordinates. When two halos merge, the galaxy of the most massive halo "jumps" onto the central particle of the resulting halo. The other galaxy still retains its identity with its original dark 
matter particle, and becomes a "satellite" within the new halo. This approach makes the trajectory of galaxies in phase space discontinous, but it assures a smooth evolution of the luminosity and stellar mass of the galaxies.

We then implement the semi-analytic technique as follows. We assume that the gas is in hydrostatic equilibrium within the dark matter halo. We model both the baryonic and the dark matter components with truncated isothermal spheres. The gas cools and flows on to the central particle of the halo instantaneously, if the cooling time is shorter than the Hubble time at that epoch, or at a given rate, otherwise.

The stellar mass $M_{*}$ increases accordingly to

$$
\frac{d M_{*}}{d t}=\alpha M_{\text {cold }} \times 10 \frac{V_{c}}{R_{\text {vir }}},
$$

where $M_{\text {cold }}$ is the mass of cold gas, $R_{\text {vir }}$ is the radius of the halo of mass $M_{\text {vir }}$, and $V_{c}^{2}=G M_{\mathrm{vir}} / R_{\mathrm{vir}}$ is the halo circular velocity. The star formation efficiency $\alpha$ is a free parameter.

Given the initial mass function for the stellar population (Scalo 1986), we can compute the number $\eta_{S N}$ of supernovae of Type II expected per unit mass. We neglect supernovae of Type Ia. A fraction $\epsilon$, kept as a free parameter, of the kinetic energy $E_{S N}$ ejected by each supernova reheats part of the cold gas to the halo virial temperature. The mass of the reheated gas over the time $\Delta t$ is

$$
\Delta M_{\text {reheat }}=\epsilon \frac{d M_{*}}{d t} \frac{4 \eta_{S N} E_{S N}}{3 V_{c}^{2}} \Delta t .
$$

Satellites merge with the central galaxy on a timescale set by dynamical friction. We compute this timescale analytically. Our prescription does not allow for merging between satellites. This shortcoming is partly responsible for the overproduction of central bright galaxies, along with the fact that we do not implement a model of chemical evolution (e.g. Kauffmann \& Charlot 1998), which affects the gas cooling rate (see Section 3). Luminosity evolution in different bands is computed with stellar population synthesis models (Bruzual \& Charlot 1999). Finally, galaxy luminosities are dimmed with an empirical prescription for dust extinction (Cardelli et al. 1989).

We apply our technique to two variants of a CDM Universe: a $\tau \mathrm{CDM}$ and a $\Lambda \mathrm{CDM}$ Universe. A complete description of these models and our technique is in Kauffmann et al. (1999a).

\section{The Luminosity Function of Galaxy Systems}

A scientific theory is required to provide testable predictions. Sometimes, it also automatically provides explanations for phenomena for which the theory was not originally constructed.

For example, consider the luminosity function of galaxies within different environments. It is well known that the usual Schechter function (Schechter 1976) does not fit all galaxy samples well. Many clusters (e.g. Biviano et al. 1995; Molinari et al. 1998; Trentham 1998), rich groups (Koranyi et al. 1998) and even compact groups (Hunsberger et al. 1998; see, however, e.g. de Carvalho 


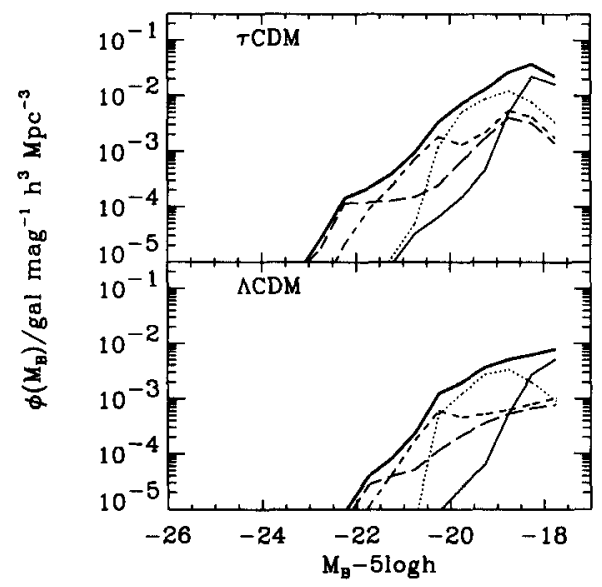

Figure 1. Blue-band luminosity function of galaxies within systems of different total mass: long-dashed line, $M>10^{14} h^{-1} M_{\odot}$; short-dashed line, $10^{13} h^{-1} M_{\odot}<M \leq 10^{14} h^{-1} M_{\odot} ;$ dotted line, $10^{12} h^{-1} M_{\odot}<M \leq 10^{13} h^{-1} M_{\odot} ;$ thin solid line, $M \leq 10^{12} h^{-1} M_{\odot}$. The bold solid line is the total luminosity function.

et al. 1994 and Zepf et al. 1997) show a two component luminosity function: a roughly Gaussian distribution at the bright end, superimposed on a Schechter function which dominates at the faint end. Because ellipticals and S0's are generally more luminous than spirals and irregulars, the Gaussian component is mostly populated by early type galaxies, and the Schechter component by late type galaxies. Moreover, because this bump at the bright end peaks at different magnitudes depending on the system considered, when we consider a large galaxy sample, which includes different environments, this feature disappears, and a Schechter function provides a reasonably good fit (e.g. Marzke et al. 1994).

Simulations of the formation of groups and clusters, where galaxies are resolved and are allowed to merge, predict a typical two component mass function at later times. This result has been well known since very early $N$-body simulations (Aarseth \& Fall 1980; Roos \& Aarseth 1982: Cavaliere et al. 1991). Thus, on the assumption that light is proportional to mass, galaxy merging provides a natural explanation for the bump observed in the luminosity function of galaxy systems. The fact that this bump is not always present clearly contains information on the formation history of the galaxy system.

Our models, designed to reproduce the global properties of galaxies and their large scale distribution rather than the luminosity function of individual clusters, also show this characteristic bump for massive systems (long and short dashed lines in Fig. 1).

Apparently, the global luminosity function (bold solid line) shows no bump. Note that the global luminosity function is not a Schechter function. To obtain 


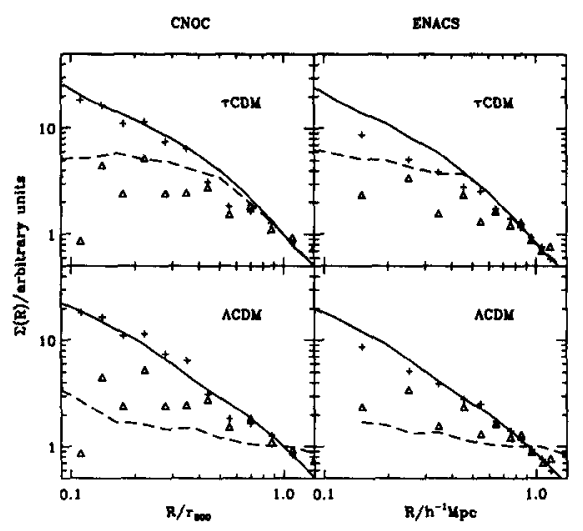

Figure 2. Surface number density profiles of galaxies within clusters of mass $M>10^{14} h^{-1} M_{\odot}$ compared with observed cluster samples. Solid (dashed) lines show the profiles for red (blue) galaxies in the models. Crosses and triangles are for red and blue galaxies in the CNOC sample (left panels) and for non-emission-line galaxies and emissionline galaxies for the ENACS sample (right panels).

the Schechter form, it is sufficient to use a dynamical friction prescription derived from high resolution $N$-body simulations (Springel et al. 1999), which automatically includes merging between satellites (Somerville \& Primack 1999), rather than our analytic prescription (Section 2). The agreement with the Schechter function can be improved further by modelling the chemical enrichment of the interstellar medium, which reduces the cooling efficiency at early times.

\section{Morphology-Density Relation and Velocity Bias}

The morphology-density relation has been known since the very beginning of the systematic investigation of galaxies (e.g. Hubble \& Humason 1931). It has also been known that late type galaxies within clusters have larger velocity dispersion than early type galaxies (e.g. Moss \& Dickens 1977). Modelling galaxy and large scale structure formation at the same time provides a self-consistent way of explaining these properties of cluster galaxies.

Fig. 2 compares our models with the surface number density profiles of galaxy clusters. Profiles are for blue and red galaxies (CNOC sample, Carlberg et al. 1997) and galaxies with emission and non-emission line spectra (ENACS, Biviano et al. 1997). The agreement is satisfactory. In fact, the differences between models and observations are simply due to the galaxy subsample definition: real galaxies have been selected according to their spectral properties which we do not model here. 


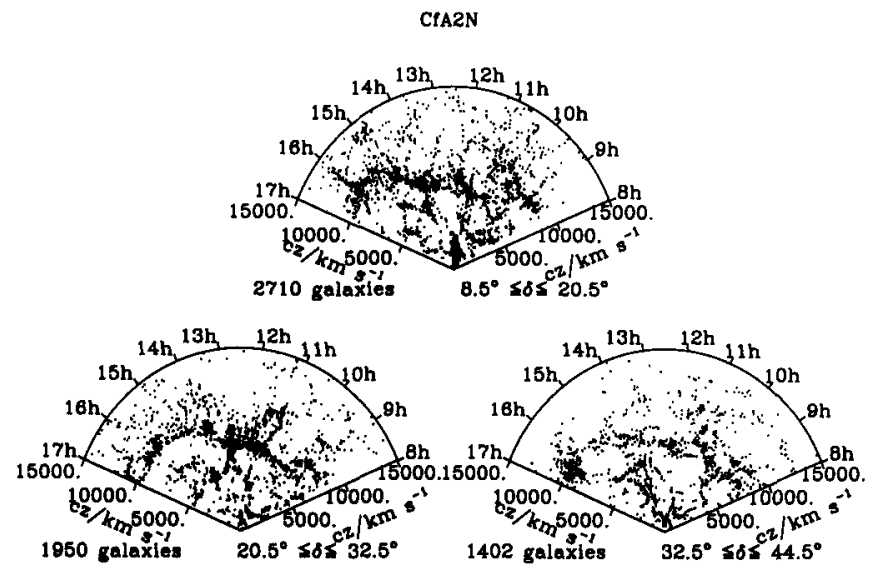

Figure 3. Galaxy distribution in the CfA2N catalogue projected on to three declination intervals.

The models can also account for the larger velocity dispersion of blue galaxies compared to red galaxies. Blue galaxies are falling on to the cluster for the first time and will become red later, when they have exhausted their cold gas reservoirs. Although this plausible explanation is well-known (e.g. Mohr et al. 1996), our models yield this result self-consistently. Moreover, our models show how this result depends on the cosmological model (Diaferio et al. 1999, Diaferio 1999).

Our technique is therefore particularly promising. For example, when simulations with better spatial and force resolution become available, it will be possible to investigate those kinematic properties of compact group members which, at present, seem difficult to interpret. For example, high velocity galaxies (Sulentic, these proceedings) may simply be due to favourable, albeit rare, conditions of infall from the surroundings (Diaferio et al. 1994).

\section{Mock Redshift Surveys}

Our simulation boxes have volumes that are comparable to the volume probed by the northern region of the Center for Astrophysics magnitude limited Redshift Survey (CfA2N hereafter; Geller \& Huchra 1989; Huchra et al. 1990; de Lapparent et al. 1991; Huchra et al. 1995; Falco et al. 1999). Therefore, we are able to compare our models with a large portion of the Universe by analysing our simulation box the same way observers analyse their data. 


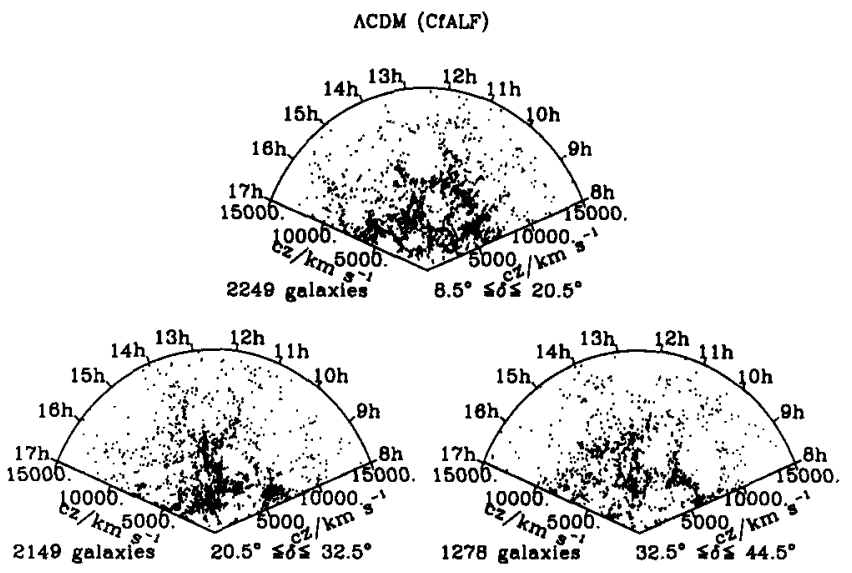

Figure 4. A mock catalogue extracted from the $\Lambda$ CDM simulation box.

We have compiled mock redshift surveys in order to have a cluster as massive as Coma at the same location as in the CfA2N (see Diaferio et al. 1999 for details). Fig. 3 shows the CfA2N and Fig. 4 shows a mock catalogue from one of our simulation boxes. The large scale structure in our simulation is not as sharply defined as in the real Universe: for example no Great Wall (Geller \& Huchra 1989 ) is present in the model. Schmalzing \& Diaferio (1999) have quantified these topological differences on large scale using Minkowski functionals.

Further constraints on the model of galaxy and structure formation come from clustering on smaller scales, in particular from the properties of galaxy groups (Ramella et al. 1997, 1999; Tucker, these proceedings).

Catalogues of groups are usually compiled using a friends-of-friends algorithm on redshift data (Huchra \& Geller 1982). However, in these redshift surveys, we do not know the true distance to a galaxy; we only know its redshift, which also includes the galaxy peculiar velocity. Therefore, the average properties of groups extracted from a redshift survey do not necessarily agree with those of groups extracted from an ideal survey in configuration space.

$N$-body simulations allow us to quantify this difference. Although previous analyses suggest that the differences are small (e.g. Nolthenius \& White 1987; Moore et al. 1993; Frederic 1995a, 1995b; Nolthenius et al. 1997), these analyses do not include the physics of galaxy formation.

On the other hand, we are able to compile redshift surveys that take into account the luminosities of galaxies, which result from their merging and stellar evolution histories. We find that typical parameters of the friends-of-friends algorithm yield kinematic properties which are in agreement with both those of 


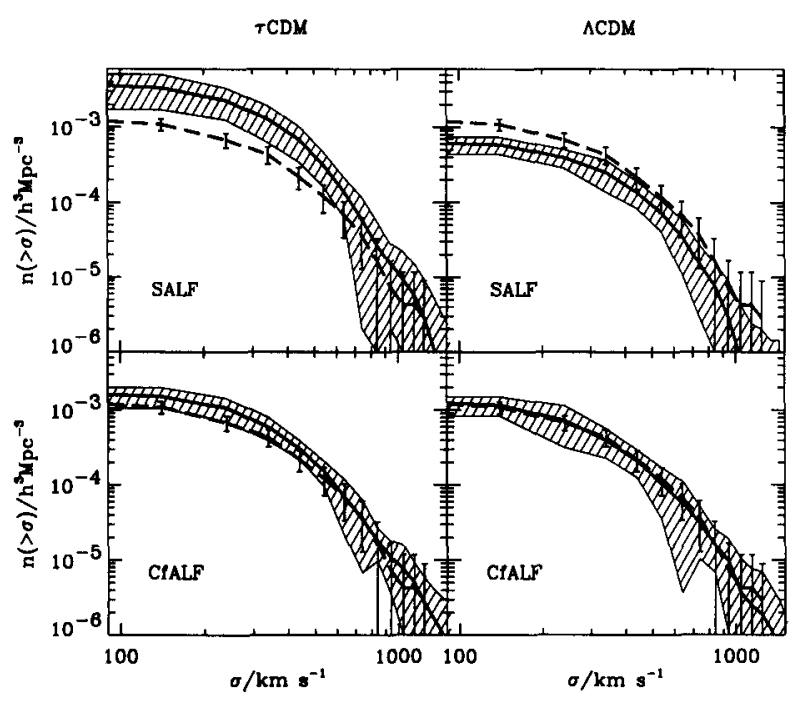

Figure 5. Group abundance by velocity dispersion $\sigma$. Bold lines are the mean number densities averaged over an ensamble of ten mock catalogues. Shaded areas show the 3- $\sigma$ deviations. Dashed lines are for the CfA2N groups. Error bars on the CfA2N curves are Poisson 3- $\sigma$ deviations. Top and bottom panels are for two different model luminosity functions (see Diaferio et al. 1999).

groups selected from configuration space and those of the CfA2N groups (Fig. 5 ). However, $40 \%$ of the triplets selected in redshift space are, in fact, unrelated galaxies which are not bound systems in configuration space.

Some of the differences between groups in the models and in the CfA2N is due to the differing distribution of galaxies on large scale. An $N$-body simulation constrained to have the same large scale structure as the local Universe (Mathis et al. 1999) will be valuable for further constraining the galaxy formation recipes using the properties of groups.

\section{Conclusion}

Combining semi-analytic modelling with $N$-body simulations is an extremely powerful tool for investigating galaxy and group formation within a self-consistent cosmological framework. $N$-body simulations with better spatial and force resolution than the simulations presented here will be ideal for understanding the properties of compact groups, their different morphology and environment (e.g. de Carvalho, these proceedings), their abundance in the local Universe and at high redshift. 
Groups selected objectively from two-dimensional information (Prandoni et al. 1994), with follow-up redshift measurements (Iovino, these proceedings), or directly selected in redshift space (Barton et al. 1996; Allam, these proceedings), can provide a wealth of information on both cosmology and galaxy formation processes.

The physics of the intergalactic medium should also be included in our approach (Cavaliere et al. 1998; Cavaliere, these proceedings): both H I (e.g. Combes; Verdes-Montenegro; Sancisi, these proceedings) and X-ray observations (e.g. Mulchaey; Ponman, these proceedings) can show clear signatures of galaxy interactions and tell us about the dynamical state of the group (Diaferio et al. 1995).

To simulate the evolution of a group of galaxies, we can extract the initial conditions and the external tidal field from a large-scale $N$-body simulation. This strategy has already been extremely successful in simulating galaxy clusters (Tormen et al. 1997; Springel et al. 1999). Thus, high-resolution $N$ body/hydrodynamic simulations will enable us to predict the properties of the galaxies and the intergalactic medium of individual groups within a full cosmological context.

Acknowledgments. The $N$-body simulations were carried out at the Computer Center of the Max-Planck Society in Garching and at the EPPC in Edinburgh, as part of the Virgo Consortium project. I thank Jörg Colberg, Guinevere Kauffmann and Simon White for permission to reproduce results from our joint research programme within the German Israeli Foundation (GIF) collaboration. I thank Margaret Geller and John Huchra for allowing the comparison of the CfA2N data directly with the simulations, and Ravi Sheth for a careful reading of the manuscript. I thank Mauri Valtonen for the invitation to this meeting, and him and the other organizers for a stimulating conference and a delightful time in Turku. Special thanks to Reinaldo de Carvalho and Giorgio Palumbo for lovely discussions and unexpected lightening endings of some Finnish nights.

\section{References}

Aarseth, S. J., \& Fall, S. M. 1980, ApJ, 236, 43

Athanassoula, E., Makino, J., \& Bosma, A. 1997, MNRAS, 286, 825

Barnes, J. E. 1985, MNRAS, 215, 517

Barton, E. J., Geller, M. J., Ramella, M., Marzke, R. O., \& da Costa, L. N. 1996, AJ, 112, 871

Biviano, A., et al., 1995, A\&A, 297, 610

Biviano, A., Katgert, P., Mazure, A., Moles, M., den Hartog, R., Perea, J., \& Focardi, P. 1997, A\&A, 321, 84

Bower, R. J. 1991, MNRAS, 248, 332

Bruzual, A., \& Charlot, S. 1999, in preparation

Cardelli, J. A., Clayton, G. C., \& Mathis, J. S. 1989, ApJ, 345, 245

Carlberg, R. G., et al., 1997, ApJ, 476, L7

Carnevali, P., Cavaliere, A., \& Santangelo, P. 1981, ApJ, 249, 449

Cavaliere, A., Colafrancesco, S., \& Menci, N. 1991, ApJ, 376, L37 
Cavaliere, A., Menci, N., \& Tozzi, P. 1998, ApJ, 501, 493

de Carvalho, R. R., Ribeiro, A. L. B., \& Zepf, S. E. 1994, ApJS, 93, 47

de Lapparent, V., Geller, M. J., \& Huchra, J. P. 1991, ApJ, 369, 273

Diaferio, A. 1999, MNRAS, in press

Diaferio, A., Geller, M. J., \& Ramella, M. 1994, AJ, 107, 868

Diaferio, A., Geller, M. J., \& Ramella, M. 1995, AJ, 109, 2293

Diaferio, A., Kauffmann, G., Colberg, J. M., \& White, S. D. M. 1999, MNRAS, 307,537

Diaferio, A., Ramella, M., Geller, M. J., \& Ferrari, A. 1993, AJ, 105, 2035

Efstathiou, G., Bond, J. R., \& White, S. D. M. 1992, MNRAS, 258, 1P

Falco, E. E., et al., 1999, PASP, 111, 438

Frederic, J. J. 1995a, ApJS, 97, 259

Frederic, J. J. 1995b, ApJS, 97, 275

Geller, M. J., \& Huchra, J. P. 1989, Science, 246, 897

Hernquist, L., Katz, N., \& Weinberg, D. H. 1995, ApJ, 442, 57

Hickson, P. 1997, ARA\&A, 35, 357

Hubble, E., \& Humason, M. L. 1931, ApJ, 74, 43

Huchra, J. P., \& Geller, M. J. 1982, ApJ, 257, 423

Huchra, J. P., de Lapparent, V., Geller, M. J., \& Corwin, Jr., H. G. 1990, ApJS, 72,433

Huchra, J. P., Geller, M. J., \& Corwin, Jr., H. G. 1995, ApJS, 99, 391

Hunsberger, S. D., Charlton, J. C., \& Zaritsky, D. 1998, ApJ, 505, 536

Kauffmann, G., \& Charlot, S. 1998, MNRAS, 294, 705

Kauffmann, G., Colberg, J. M., Diaferio, A., \& White, S. D. M. 1999a, MNRAS, 303,188

Kauffmann, G., Colberg, J. M., Diaferio, A., \& White, S. D. M. 1999b, MNRAS, 307,529

Koranyi, D., Geller, M. J., Mohr, J. J., \& Wegner, G. 1998, AJ, 116, 2108

Mamon, G. A. 1986, ApJ, 307, 426

Mamon, G. A. 1989, A\&A, 219, 98

Marzke, R. O., Huchra, J. P., \& Geller, M. J. 1994, ApJ, 428, 43

Mathis, H., et al. (the GIF collaboration), 1999, in preparation

Mazure, A., \& Le Fèvre, O. 1999, Proceedings of the IGRAP International Conference, Clustering at High Redshift, Marseilles, $29^{\text {th }}$ June- $2^{\text {nd }}$ July 1999 , in press

Mohr, J. J., Geller, M. J., Fabricant, D. G., Wegner, G., Thorstensen, J., \& Richstone, D. O. 1996, ApJ, 470, 724

Molinari, E., Chincarini, G., Moretti, A., \& de Grandi, S. 1998, A\&A, 338, 874

Moore, B., Frenk, C. S., \& White, S. D. M. 1993, MNRAS, 261, 827

Moss, C., \& Dickens, R. J. 1977, MNRAS, 178, 701

Nolthenius, R. A., \& White, S. D. M. 1987, MNRAS, 235, 505

Nolthenius, R. A., Klypin, A. A., \& Primack, J. R. 1997, ApJ, 480, 43 
Peacock, J. A. 1999, Cosmological Physics, Cambridge University Press, Cambridge

Pearce, F. R., et al., 1999, ApJ, 512, L99

Prandoni, I., Iovino, A., \& MacGillivray, H. T. 1994, AJ, 107, 1235

Press, W. H., \& Schechter, P. 1974, ApJ, 187, 425

Ramella, M., et al., 1999, A\&A, 342, 1

Ramella, M., Pisani, A., \& Geller, M. J. 1997, AJ, 113, 483

Roos, N., \& Aarseth, S. J. 1982, A\&A, 114, 41

Scalo, J. M. 1986, Fundamentals of Cosmic Physics, 11, 3

Schechter, P. 1976, ApJ, 203, 297

Schmalzing, J., \& Diaferio, A. 1999, MNRAS, submitted

Somerville, R. S., \& Primack, J. R., 1999, MNRAS, submitted (astro-ph/9802268)

Springel, V., White, S. D. M., Tormen, G., \& Kauffmann, G. 1999, in preparation

Tormen, G., Bouchet, F. R., \& White, S. D. M. 1997, MNRAS, 286, 865

Trentham, N. 1998, MNRAS, 294, 193

Zepf, S. E., de Carvalho, R. R., \& Ribeiro, A. L. B. 1997, ApJ, 488, L11 